JOURNAL OF INTEGRAL EQUATIONS AND APPLICATIONS

Volume 6, Number 2, Spring 1994

\title{
DISCRETE POLYNOMIAL-BASED GALERKIN METHODS FOR FREDHOLM INTEGRAL EQUATIONS
}

\author{
MICHAEL A. GOLBERG
}

1. Introduction. In recent years there has been considerable interest in using Galerkin's method for the numerical solution of Fredholm integral equations. In large measure, this interest seems to stem from the interesting superconvergence properties discovered by Sloan in $[\mathbf{1 2}$, 13]. In early work the effect of quadrature errors on the behavior of the algorithms was ignored $[\mathbf{1 2}, \mathbf{1 3}]$, but starting with the work of Chandler [4] and then Spence and Thomas [14], these errors have been studied in great detail. For spline approximations this work culminated in the papers of Joe [7] and Atkinson and Bogomolny [2]. In particular, in [2], it was shown that sufficiently accurate quadrature rules preserved both the rates of convergence and superconvergence of Galerkin's method.

In [5] Delves and Freeman discussed the effect of quadrature errors on Galerkin's method using orthogonal polynomial approximations for one-dimensional equations, while Miel in $[\mathbf{1 0}, \mathbf{1 1}]$ examined the particular case of Legendre polynomial approximations for both linear and nonlinear equations. None of these authors considered the convergence of the Sloan iterate.

It is the purpose of this paper, therefore, to sharpen and extend the convergence results in $[\mathbf{5}, \mathbf{1 0}, \mathbf{1 1}]$ for the solution of the equations

$$
u(x)=f(x)+\int_{a}^{b} k(x, t) u(t) d t, \quad-\infty<a<b<\infty,
$$

where $f(x)$ and $k(x, t)$ are suitably smooth functions on $[a, b]$ and $[a, b] \times$ $[a, b]$ respectively. In particular, we show that if $u(x)$ is approximated by $v_{n}=\sum_{k=0}^{n} a_{k} \varphi_{k}(x)$, where $\left\{\varphi_{n}\right\}$ are the orthonormal polynomials associated with the integrable weight function $w(x) \geq 0$ on $[a, b]$ and integration rules of precision greater or equal than $2 n$ are used to evaluate the integral transforms and inner products, then $\| u-$

\footnotetext{
Received by the editors on January 21,1993 , and in revised form on January 13, 1994.

Copyright (C)1994 Rocky Mountain Mathematics Consortium
} 
$v_{n} \|_{\infty}=O\left(n^{-r+\nu+\mu+2}\right)$ where $r$ is the number of derivatives of $f(x)$ and $k(x, t)$ and $\nu$ and $\mu$ depend on $\left\{\varphi_{n}\right\}$. This extends the mean-square convergence results in $[\mathbf{5}, \mathbf{1 0}, \mathbf{1 1}]$.

The paper is divided into five sections. In Sections 2 and 3, we review the Galerkin and discrete Galerkin methods. Section 4 is devoted to the convergence analysis of the discrete Galerkin method. In Section 5 we summarize these results and discuss future research.

2. Galerkin's method. Assume that $k(x, t)$ and $f(x)$ in (1.1) are real and continuous on $[a, b],-\infty<a<b<\infty$, and let $L_{w}$ be the space of real square-integrable functions with respect to the integrable weight function $w(x) \geq 0$. The inner product on $L_{w}$ is given by

$$
\langle f, g\rangle_{w}=\int_{a}^{b} w(x) f(x) g(x) d x
$$

and the induced norm is

$$
\|f\|_{w}=\left(\langle f, f\rangle_{w}\right)^{1 / 2} .
$$

To solve (1.1) (which is assumed to have a unique solution) by Galerkin's method, let $\left\{\varphi_{0}, \ldots, \varphi_{n}\right\}$ be $n+1$ linearly independent functions in $L_{w}$ and approximate $u$ by

$$
u_{n}=\sum_{k=0}^{n} a_{k} \varphi_{k} .
$$

As is well known, the coefficients $\left\{a_{k}\right\}_{k=0}^{n}$ are obtained by solving [1]

$$
\begin{gathered}
\left(K \varphi_{k}=\int_{a}^{b} k(x, t) \varphi_{k}(t) d t\right) \\
\sum_{k=0}^{n}\left\langle\varphi_{k}, \varphi_{j}\right\rangle_{w} a_{k}-\sum_{k=0}^{n}\left\langle K \varphi_{k}, \varphi_{j}\right\rangle_{w} a_{k}=\left\langle f, \varphi_{j}\right\rangle_{w}, \\
j=0,1,2, \ldots, n .
\end{gathered}
$$

We shall consider equations $(2.3)-(2.4)$ only where $\left\{\varphi_{0}, \ldots \varphi_{n}\right\}, n=$ $0,1,2, \ldots$, are the orthonormal polynomials associated with $w(x)$. That is,

(i) $\operatorname{deg}\left(\varphi_{n}\right)=n, \quad n=0,1,2, \ldots$,

(ii) $\left\langle\varphi, \varphi_{j}\right\rangle_{w}=\delta_{k j}, \quad(k, j)=0,1,2, \ldots, n$. 
Particularly important special cases occur when $w(x)=1 /\left(1-x^{2}\right)^{1 / 2}$ and $\varphi_{n}(x)=t_{n}(x)$, the $n$th orthonormalized Chebyshev polynomial [3], or $w(x)=1$ and $\varphi_{n}=p_{n}$, the normalized Legendre polynomials [10].

Under the stated conditions on $k$ and $f$, it is well known that $u_{n}$ converges to $u$ in $L_{w}[\mathbf{1 2}]$. In particular, using Jackson's theorem,

$$
\left\|u-u_{n}\right\|_{w}=0\left(n^{-r}\right)
$$

if $f(x) \in C^{r}[a, b]$, and $k(x, t) \in C^{r}([a, b] \times[a, b])$. For some polynomials one can obtain convergence rates in the uniform norm [3].

The convergence property in (2.7) has been known for some time and has been proved under the assumption that the inner products $\left\langle f, \varphi_{j}\right\rangle_{w},\left\langle K \varphi_{k}, \varphi_{j}\right\rangle_{w},(j, k)=0,1,2, \ldots, n$, are calculated exactly. In most practical cases, this cannot be done, and some numerical method needs to be used to approximate them. It is then important to study the effects of such approximations on the convergence of $u_{n}$. For piecewise polynomials (splines) the work of Chandler [4], Spence and Thomas [14], Joe [7], and Atkinson and Bogomolny [2] has shown that sufficiently accurate quadrature rules will preserve the convergence rates of the Galerkin approximation (they require $w=1$ ). In particular, using discontinuous splines of order $r[r=\operatorname{deg}+1]$, Atkinson and Bogomolny have shown that, to preserve the $O\left(h^{r}\right)$ rate of convergence of Galerkin's method, one must use an integration rule with precision $d=r-1$. To preserve the $O\left(h^{2 r}\right)$ convergence of the Sloan iterate, it suffices to use an integration rule with $d \geq 2 r-1$. On the basis of this, it is reasonable to conjecture that rules of precision $n$ could be used to preserve (2.7). Unfortunately, this appears not to be the case. The best we are able to show is that rules with precision $\geq 2 n$ give $O\left(n^{-r+\nu+\mu+2}\right)$ convergence in the uniform norm $(\nu \geq 0, \mu \geq 0$ depend on $\left.\left\{\varphi_{n}\right\}_{n=0}^{\infty}\right)$ while Gaussian quadrature with $n+1$ nodes and $w(x)=1$ gives $O\left(n^{-r}\right)$ convergence in the $L_{w}$ norm.

3. Discrete Galerkin methods. When the inner products and integrals in (2.4) are approximated by numerical integration, the approximation to $u_{n}$ will be denoted by $v_{n}$ and the resulting numerical scheme will be referred to as the discrete Galerkin method. 
To obtain $v_{n}$, define quadrature rules $Q_{M}$ and $Q_{N}$ by

$$
\int_{a}^{b} w(x) g(x) d x \simeq Q_{M}(g)=\sum_{k=0}^{M(n)} w_{k} g\left(x_{k}\right),
$$

and

$$
\int_{a}^{b} g(t) d t \simeq Q_{N}(g)=\sum_{l=0}^{N(n)} \sigma_{l} g\left(t_{l}\right) .
$$

(Note that $\left\{w_{k}\right\},\left\{x_{k}\right\},\left\{\sigma_{l}\right\}$ and $\left\{t_{l}\right\}$ generally depend on $n$. For convenience, this dependence will be suppressed in the remainder of the paper.) For our purposes we require that

(i) $w_{k}>0, \quad k=0,1,2, \ldots, M(n), n \geq 0$

(ii) $\sigma_{l}>0, \quad l=0,1,2, \ldots, N(n), n \geq 0$,

(iii) the precision of $Q_{N}$ and $Q_{M}$ is $\geq 2 n, \quad n \geq 0$.

That is,

$$
Q_{M}(g)=\int_{a}^{b} w(x) g(x) d x, \quad Q_{N}(g)=\int_{a}^{b} g(x) d x,
$$

when $g$ is a polynomial of degree $\leq 2 n$.

Using $Q_{M}$ and $Q_{N}$ we define the following approximations:

$$
\begin{aligned}
\left\langle f, \varphi_{k}\right\rangle_{w} & \simeq Q_{M}\left(f \varphi_{k}\right), \quad 0 \leq k \leq n, \\
\left\langle\varphi_{k}, \varphi_{j}\right\rangle_{w} & \simeq Q_{M}\left(\varphi_{k} \varphi_{j}\right), \quad 0 \leq(j, k) \leq n
\end{aligned}
$$

and

$$
\begin{aligned}
\left\langle K \varphi_{k}, \varphi_{j}\right\rangle_{w} & =\int_{a}^{b} \int_{a}^{b} w(x) k(x, t) \varphi_{k}(t) \varphi_{j}(x) d x d t \\
& \simeq \sum_{m=0}^{M} \sum_{l=0}^{N} \sigma_{l} w_{m} k\left(x_{m}, t_{l}\right) \varphi_{k}\left(t_{l}\right) \varphi_{j}\left(x_{m}\right) \\
& \equiv Q_{M} \times Q_{N}\left(k \varphi_{k} \varphi_{j}\right) .
\end{aligned}
$$


Substituting (3.6)-(3.8) into (2.6) and letting

$$
v_{n}=\sum_{k=0}^{n} b_{k} \varphi_{k}
$$

$\left\{b_{k}\right\}_{k=0}^{n}$ are determined by solving

$$
\begin{aligned}
b_{j}-\sum_{k=0}^{n}\left[\sum_{m=0}^{M} \sum_{l=0}^{N} \sigma_{l} w_{m} k\left(x_{m}, t_{l}\right) \varphi_{k}\left(t_{l}\right) \varphi_{j}\left(x_{m}\right)\right] \\
=\sum_{m=0}^{M} w_{m} f\left(x_{m}\right) \varphi_{j}\left(x_{m}\right), \quad 0 \leq j \leq n
\end{aligned}
$$

since $Q_{N}$ has precision $\geq 2 n$ and $\varphi_{k} \varphi_{j}$ is a polynomial of degree $j+k \leq 2 n, 0 \leq(j, k) \leq n$ so that $Q_{M}\left(\varphi_{k} \varphi_{j}\right)=\left\langle\varphi_{k}, \varphi_{j}\right\rangle_{w}=\delta_{k j}$.

\section{Convergence of the discrete Galerkin method}

4.1. Mean-square convergence of $v_{n}$. To prove the convergence of $v_{n}$ and to obtain rates of convergence, we use the theory of perturbed projection methods $[\mathbf{8}]$. For this, let $P_{n}$ be the operator of orthogonal projection onto $X_{n}=\operatorname{span}\left(\left\{\varphi_{k}\right\}_{k=0}^{n}\right)$. Then some tedious algebra shows that $v_{n}$ defined by $(3.9)-(3.10)$ satisfies the operator equation

$$
v_{n}=\pi_{n} K_{n} v_{n}+\pi_{n} f
$$

where

$$
K_{n} u(x)=\sum_{l=0}^{N(n)} \sigma_{l} k\left(x, t_{l}\right) u\left(t_{l}\right)
$$

and $\pi_{n}$ is a "discrete" projection defined by

$$
\pi_{n} u(x)=\sum_{k=0}^{n} Q_{M}\left(u \varphi_{k}\right) \varphi_{k} .
$$

Now $\pi_{n} K_{n} v_{n}=P_{n} K v_{n}+\pi_{n} K_{n} v_{n}-P_{n} K v_{n}=P_{n} K v_{n}+R_{n} v_{n}$ and $\pi_{n} f=\pi_{n} f-P_{n} f+P_{n} f=P_{n} f+r_{n}$ so that (4.1) becomes

$$
v_{n}=P_{n} K v_{n}+R_{n} v_{n}+P_{n} f+r_{n},
$$


where $R_{n}$ may be viewed as a linear operator from $X_{n} \rightarrow X_{n}, r_{n} \in X_{n}$ and $v_{n} \in X_{n}$.

Let

$$
\left\|R_{n}\right\|_{n}=\left\{l u b|| R_{n} w_{n}\left\|_{w}, w_{n} \in X_{n},\right\| w_{n} \|=1\right\} .
$$

Theorem 4.1. Let $Q_{M}$ and $Q_{N}, n \geq 0$, be a sequence of quadrature rules satisfying (3.3)-(3.5), and assume that $f(x) \in C^{r}[a, b], r>\nu+1$, $k(x, t) \in C^{r}([a, b] \times[a, b]), r>\nu+1$, and $\left[\int_{a}^{b} \varphi_{n}^{2}(x) d x\right]^{1 / 2} \leq c n^{\nu}, \nu \geq 0$, $n \geq 0$ where $c$ does not depend on $n$. Then for all $n$ sufficiently large, $v_{n}$, the discrete Galerkin approximation to $u$, exists and is unique in $L_{w}$. Moreover, $v_{n} \rightarrow u$ in $L_{w}$ and

$$
\left\|u-v_{n}\right\|_{w}=0\left(n^{-r+\nu+1}\right) .
$$

To prove Theorem 4.1 we need to obtain estimates of the quadrature errors

$$
e_{j}\left(f \varphi_{j}\right)=\left\langle f, \varphi_{j}\right\rangle_{w}-Q_{M}\left(f \varphi_{j}\right), \quad 0 \leq j \leq n
$$

and

$$
E_{j k}=\left\langle K \varphi_{k}, \varphi_{j}\right\rangle_{w}-Q_{M} \times Q_{N}\left(k \varphi_{k} \varphi_{j}\right), \quad 0 \leq(j, k) \leq n .
$$

These estimates are the core of the proof, and our method for obtaining them is somewhat different than that used in $[\mathbf{5}, \mathbf{6}]$. Similar results to these are given in $[\mathbf{9}]$, but it appears from the proof given there that the constants in (4.9)-(4.10) depend on the derivatives of $\left\{\varphi_{n}\right\}_{n=0}^{\infty}$. Since these derivatives can grow like $n^{r}$, where $r$ is the number of derivatives of $k(x, t)$ and $f(x)$, this growth shows that the errors need not converge to zero. The convergence rates are an improvement over those given in $[\mathbf{5}, \mathbf{1 0}]$. We begin with a simple, but useful lemma.

Lemma 4.1. Let $X$ be a normed linear space with norm $\|\cdot\|$, and let $l$ be a bounded linear functional on $X$. If $Y$ is a subspace of $X$ and $l(y)=0$ for all $y \in Y$, then for all $x \in X$,

$$
|l(x)| \leq\|l\|\{\inf \|x-y\|, y \in Y\} .
$$


Proof. Let $x \in X$. Then $x=(x-y)+y, y \in Y$. Since $l$ is linear and $l(y)=0, l(x)=l(x-y)+l(y)=l(x-y)$. Taking absolute values gives (4.8).

Lemma 4.2. Let $Q_{M}$ and $Q_{N}$ satisfy (3.3)-(3.5), and let $g(x) \in$ $C^{r}[a, b], r \geq 1$. Assume for some $\nu \geq 0$,

$$
\left[\int_{a}^{b} \varphi_{j}^{2}(x) d x\right]^{1 / 2} \leq c j^{\nu}
$$

with $c$ independent of $j$. Then

$$
\begin{aligned}
\left|e_{j}(g)\right| & =\left|\int_{a}^{b} w(x) g(x) \varphi_{j}(x) d x-Q_{M}\left(g \varphi_{j}\right)\right| \\
& \leq c / n^{r}, \quad 0 \leq j \leq n,
\end{aligned}
$$

and

$$
\begin{aligned}
\left|f_{j}(g)\right| & =\left|\int_{a}^{b} g(x) \varphi_{j}(x) d x-Q_{N}\left(g \varphi_{j}\right)\right| \\
& \leq c / n^{r-\nu}, \quad 0 \leq j \leq n,
\end{aligned}
$$

where $c$ is a generic constant not depending on $n$.

Proof. To get (4.9), take $X=C[a, b]$ with the sup norm, in Lemma 4.1, and let $Y=X_{n}=\operatorname{span}\left(\left\{\varphi_{k}\right\}_{k=0}^{n}\right)$, the subspace of polynomials of degree $\leq n$. If $l_{j}=e_{j}$, then $l_{j}(g)=0$, for all $g \in X_{n}$, since $Q_{N}$ has precision $\geq 2 n$. Thus, if $g \in C^{r}[a, b]$, $\left|e_{j}(g)\right|=\left|l_{j}(g)\right| \leq \| l_{j}|| \inf \left\{\|g-y\|_{\infty}, y \in X_{n}\right\}$. By Jackson's theorem $\inf \left\{\|g-y\|_{\infty}, y \in X_{n}\right\}=0\left(n^{-r}\right), n \geq r$, so that it remains to estimate $\left\|l_{j}\right\|$.

Now

$$
\begin{aligned}
\left|l_{j}(g)\right| & =\left|\int_{a}^{b} w(x) g(x) \varphi_{j}(x) d x-\sum_{m=0}^{M} w_{m} g\left(x_{m}\right) \varphi_{j}\left(x_{m}\right)\right| \\
& \leq \int_{a}^{b} w(x)|g(x)|\left|\varphi_{j}(x)\right| d x+\sum_{m=0}^{M} w_{m}\left|g\left(x_{m}\right)\right|\left|\varphi_{j}\left(x_{m}\right)\right|,
\end{aligned}
$$


since $w(x) \geq 0$ and $w_{m}>0,0 \leq m \leq M$. By the Cauchy-Schwarz inequality for integrals and sums,

$$
\begin{aligned}
\int_{a}^{b} w(x)|g(x)| \mid \varphi_{j} & (x) \mid d x \\
\leq & {\left[\int_{a}^{b} w(x) \varphi_{j}^{2}(x) d x\right]^{1 / 2}\left[\int_{a}^{b} w(x) g^{2}(x) d x\right]^{1 / 2} } \\
\leq & c_{1}\|g\|_{\infty},
\end{aligned}
$$

and

$$
\begin{aligned}
\sum_{m=0}^{M} w_{m}\left|g\left(x_{m}\right)\right| & \left|\varphi_{j}\left(x_{m}\right)\right| \\
& \leq\left[\sum_{m=0}^{M} w_{m} \varphi_{j}^{2}\left(x_{m}\right)\right]^{1 / 2}\left[\sum_{m=0}^{M} w_{m} g^{2}\left(x_{m}\right)\right]^{1 / 2} \\
& \leq c_{2}\|g\|_{\infty},
\end{aligned}
$$

since $\left[\sum_{m=0}^{M} w_{m} \varphi_{j}^{2}\left(x_{m}\right)\right]^{1 / 2}=\left[\int_{a}^{b} w(x) \varphi_{j}^{2}(x) d x\right]^{1 / 2}$ and $\left[\sum_{m=0}^{M} w_{m}\right]^{1 / 2}=$ $\left[\int_{a}^{b} w(x) d x\right]^{1 / 2}$.

Thus, $\left|l_{j}(g)\right| \leq\left(c_{1}+c_{2}\right)\|g\|_{\infty}$, giving $\left\|l_{j}\right\| \leq c$ where $c$ does not depend on $j, 0 \leq j \leq n$. Hence, $\left|e_{j}(g)\right|=\left|l_{j}(g)\right| \leq c n^{-r}, 0 \leq j \leq n$.

For (4.10), we again take $X=C[a, b]$ and $Y=X_{n}$ in Lemma 4.1. Letting $l_{j}(g)=\int_{a}^{b} g(x) \varphi_{j}(x) d x-Q_{N}\left(g \varphi_{j}\right), 0 \leq j \leq n, l_{j}(g)=f_{j}(g)$. Since $Q_{N}$ also has precision $\geq 2 n, l_{j}(g)=0$ for all $g \in X_{n}, 0 \leq j \leq n$, and $\left|l_{j}(g)\right| \leq c|| l_{j}|| n^{r}$. Thus, it suffices to estimate $\left\|l_{j}\right\|$.

Arguing as above, $\left\|l_{j}(g)\right\| \leq c\left[\left(\int_{a}^{b} \varphi_{j}^{2}(x) d x\right)^{1 / 2}+\sum_{l=0}^{N} \sigma_{l}^{2} \varphi_{j}\left(x_{l}\right)^{1 / 2}\right] \times$ $\|g\|_{\infty}=2 c\left(\int_{a}^{b} \varphi_{j}^{2}(x) d x\right)^{1 / 2}\|g\|_{\infty}$ because $Q_{N}$ has precision $\geq 2 n$. By assumption, $\left(\int_{a}^{b} \varphi_{j}^{2}(x) d x\right)^{1 / 2} \leq c j^{\nu}$, so that $\left\|l_{j}\right\| \leq c j^{\nu} \leq c n^{\nu}$, $0 \leq j \leq n$. Thus, $\left|f_{j}(g)\right|=\left|l_{j}(g)\right|=0\left(n^{-r+\nu}\right)$.

Lemma 4.3. Let $g(x, y) \in C^{r}([a, b] \times[a, b]), r>\nu+1$, and suppose that $\int_{a}^{b} \int_{a}^{b} w(x) g(x, y) \varphi_{k}(x) \varphi_{j}(y) d x d y$ is approximated by $Q_{M} \times$ $Q_{N}\left(g \varphi_{k} \varphi_{j}\right)$, where $Q_{N}$ and $Q_{M}$ are as in Lemma 4.2. Then the error $E_{j k}=0\left(n^{-r+\nu}\right), 0 \leq(j, k) \leq n, n \geq r$. 
Proof. By definition

$$
\begin{aligned}
E_{j k}= & \int_{a}^{b} \int_{a}^{b} w(x) g(x, y) \varphi_{k}(x) \varphi_{j}(y) \\
& -\sum_{l=0}^{N} \sum_{m=0}^{M} \sigma_{m} w_{l} g\left(x_{l}, y_{m}\right) \varphi_{k}\left(x_{l}\right) \varphi_{j}\left(y_{m}\right) .
\end{aligned}
$$

Letting $h_{m}(x)=g\left(x, y_{m}\right), 0 \leq m \leq M$, and $h(y)=\int_{a}^{b} w(x) g(x, y) \varphi_{k}(x) d x$

$$
E_{j k}=\int_{a}^{b} h(y) d y-\sum_{m=0}^{M} \sigma_{m} \varphi_{j}\left(y_{m}\right) Q_{N}\left(h_{m} \varphi_{k}\right) .
$$

But $Q_{N}\left(h_{m} \varphi_{k}\right)=\int_{a}^{b} w(x) g\left(x, y_{m}\right) \varphi_{k}(x) d x-e_{k}\left(h_{m} \varphi_{k}\right)=h\left(y_{m}\right)-$ $e_{k}\left(h_{m} \varphi_{k}\right)$, and using this in (4.14) gives

$$
\begin{aligned}
E_{j k}= & \int_{a}^{b} h(y) d y-\sum_{m=0}^{M} w_{m} \varphi_{j}\left(y_{m}\right)\left[h\left(y_{m}\right)-e_{k}\left(h_{m} \varphi_{k}\right)\right] \\
= & \int_{a}^{b} h(y) d y-\sum_{m=0}^{M} w_{m} \varphi_{j}\left(y_{m}\right) h\left(y_{m}\right) \\
& +\sum_{m=0}^{M} w_{m} \varphi_{j}\left(y_{m}\right) e_{k}\left(h_{m} \varphi_{k}\right) \\
= & f_{j}\left(h \varphi_{j}\right)-\sum_{m=0}^{M} w_{m} \varphi_{j}\left(y_{m}\right) e_{k}\left(h_{m} \varphi_{k}\right) .
\end{aligned}
$$

By Lemma $4.2,\left|f_{j}\left(h \varphi_{j}\right)\right|=0\left(n^{-r+\nu}\right), 0 \leq j \leq n$, and $\left|e_{k}\left(h_{m} \varphi_{k}\right)\right|=$ $0\left(n^{-r}\right)$ uniformly in $m$. (The uniformity follows from the error formula in Jackson's theorem [3].) Thus

$$
\left|E_{j k}\right| \leq c_{1} n^{-r+\nu}+c n^{-r} \sum_{m=0}^{M} w_{m}\left|\varphi_{j}\left(y_{m}\right)\right| .
$$

By the argument in Lemma 4.2

$$
\sum_{m=0}^{M} w_{m}\left|\varphi_{j}\left(y_{m}\right)\right| \leq\left[\int_{a}^{b} w(x) d x\right]^{1 / 2}, \quad 0 \leq j \leq n
$$


so $\left|E_{j k}\right| \leq c_{1} n^{-r+\nu}+c_{3} n^{-r} \leq c n^{-r+\nu}, 0 \leq(k, j) \leq n$.

One should note that the error estimates in Lemmas 4.2 and 4.3 do not follow directly from those for $\int_{a}^{b} w(x) g(x) d x-Q_{M}(g)$, etc., if $g$ is a $C^{r}$ function. This was apparently done in $[8]$ and leads, we believe, to over-optimistic assumptions on the quadrature rules. For example, if we assume, as is done in $[\mathbf{9}]$, that $Q_{M}$ has precision $\geq n$, then the argument given there for $e_{j}(g)$ appears to go as follows:

Let $p_{n}$ of degree $\leq n$ be the polynomial of best uniform approximation to $g \varphi_{j}$. Then

$$
\begin{aligned}
& \int_{a}^{b} w(x) g(x) \varphi_{j}(x) d x-Q_{M}\left(g \varphi_{j}\right) \\
&=\int_{a}^{b} w(x)\left[g(x) \varphi_{j}(x)-p_{n}(x)\right] d x-Q_{M}\left(g \varphi_{j}-p_{n}\right)
\end{aligned}
$$

since $\int_{a}^{b} w(x) p_{n}(x) d x-Q_{M}\left(p_{n}\right)=0$. thus,

$$
\begin{aligned}
\left|e_{j}(g)\right| \leq & \left\|g \varphi_{j}(x)-p_{n}\right\|_{\infty} \int_{a}^{b} w(x) d x \\
& +\left\|g \varphi_{j}-p_{n}\right\|_{\infty} \sum_{m=0}^{M} w_{m} \\
= & 2\left\|g \varphi_{j}-p_{n}\right\|_{\infty} \int_{a}^{b} w(x) d x .
\end{aligned}
$$

If one now uses Jackson's theorem to estimate $\left\|g \varphi_{j}-p_{n}\right\|_{\infty}$ the error is $\leq c n^{-r}\left\|\left(g \varphi_{j}\right)^{(r)}\right\|_{\infty}$ where $\left(g \varphi_{j}\right)^{(r)}$ is the $r$ th derivative of $g \varphi_{j}$. By Leibnitz's rule $\left(g \varphi_{j}\right)^{(r)}=\sum_{k=0}^{r}\left(\begin{array}{l}r \\ k\end{array}\right) \varphi_{j}^{(k)} g^{(r-k)}$ so that $\left\|g \varphi_{j}(r)\right\|_{\infty} \leq \sum_{k=0}^{r}\left(\begin{array}{c}r \\ k\end{array}\right)\left\|\varphi_{j}^{(k)}\right\|_{\infty}\left\|g^{(r-k)}\right\|_{\infty}$. But $\left\|\varphi_{j}^{(k)}\right\|_{\infty}$ can grow like $j^{2 k}$ (for Legendre or Chebyshev polynomials, for instance) so that in such cases $\left\|\left(g \varphi_{j}\right)^{(r)}\right\|_{\infty} \leq c_{1} j^{2 r}$, and it follows from this argument that $\left|e_{j}(g)\right| \leq c n^{-r} j^{2 r}$. For $j=n,\left|e_{j}(g)\right|=0\left(n^{r}\right)$, which is useless for our purposes. 
Proof of Theorem 4.1. Arguing as in [9], it follows that

$$
\left\|r_{n}\right\|_{w}=\left[\sum_{k=0}^{n} e_{k}^{2}\left(f \varphi_{k}\right)\right]^{1 / 2}
$$

and

$$
\left\|R_{n}\right\|_{n} \leq\left[\sum_{k=0}^{n} \sum_{j=0}^{n} E_{j k}^{2}\right]^{1 / 2} .
$$

Using the results of Lemmas 4.2-4.3 in (4.15)-(4.16) gives $\left\|r_{n}\right\|_{w}=$ $0\left(n^{-r+1 / 2}\right)$ and $\left\|R_{n}\right\|_{n}=0\left(n^{-r+\nu+1}\right)$, so that $\left\|r_{n}\right\|_{w} \rightarrow 0$ and $\left\|R_{n}\right\|_{n} \rightarrow 0, n \rightarrow \infty$. From Theorem 1 of [8] (let $H=I$ there) it follows that for all $n$ sufficiently large that $v_{n}$ exists, is unique, and

$$
\left\|u-v_{n}\right\|_{w} \leq c\left[\left\|u-u_{n}\right\|_{w}+\left\|R_{n}\right\|_{n}+\left\|r_{n}\right\|_{w}\right] .
$$

Since $\left\|u-u_{n}\right\|_{w}=0\left(n^{-r}\right),(4.9)-(4.10)$ and (4.17) give

$$
\left\|u-v_{n}\right\|_{w} \leq c_{1} n^{-r}+c_{2} n^{-r+\nu+1}+c_{3} n^{-r+1 / 2} \leq c n^{-r+\nu+1}, \quad n \rightarrow \infty .
$$

Example 4.1. In [16] Miel considered the discrete Galerkin method where $w(x)=1,\left\{\varphi_{n}\right\}_{n=0}^{\infty}$ are the normalized Legendre polynomials and $Q_{M}$ and $Q_{N}$ are ordinary Gaussian quadratures with $M(n)=$ $N(n)=n+1$. In this case it is well known that the precision of $Q_{M}$ and $Q_{N}$ is $2 n+1$ and all the weights $w_{m}=\sigma_{m}$ are positive [15]. If $f(x)$ and $k(t, x)$ in (1.1) are $C^{r}, r>1$, functions, it follows that the discrete Galerkin approximation $\nu_{n}$ converges in $L_{w}[a, b](w=1)$ and $\left\|u-v_{n}\right\|_{w}=0\left(n^{-r+1}\right)$ since $\nu=0$.

This sharpens the result in Theorem 5.2 of $[\mathbf{1 0}]$ where only convergence of the discrete Galerkin method is proved, but no convergence rates are given. In fact, there appears to be an error (or possibly just an oversight) in the proof, as the author states (but does not prove) that $e_{k}\left(f \varphi_{k}\right) \rightarrow 0$ and $E_{j k} \rightarrow 0$ are sufficient to guarantee that $\left\|r_{n}\right\|_{w}$ and $\left\|R_{n}\right\|_{n}$ in (4.18) also converge to zero. Since no analysis of the quadrature errors $e_{k}$ and $E_{j k}$ is given, it appears that the assumption of Riemann integrability of $f(x)$ and $k(x, t)$ is too weak. 
Example 4.2. In [3] Baker considered solving (1.1) using Galerkin's method with the Chebyshev polynomials $\left\{t_{n}(x)\right\}$. Taking $a=-1$, $b=1, w(x)=\left(1-x^{2}\right)^{-1 / 2}$, and using the Gaussian quadrature for $Q_{N}(M=N=n+1)$ gives $\left\|u-v_{n}\right\|_{w}=0\left(n^{-r+1}\right)$ as $\left\|t_{n}\right\|_{\infty} \leq 1$, $n \geq 0$.

\subsection{Uniform convergence of $v_{n}$.}

Theorem 4.2. Suppose that the conditions of Theorem 4.1 hold and $\left\|\varphi_{n}\right\|_{\infty} \leq n^{\mu}, \mu \geq 0, n \geq 0$. Then $v_{n}$ converges uniformly to $u$ if $r>\nu+\mu+2$ and

$$
\left\|u-v_{n}\right\|_{\infty} \leq n^{-r+\nu+\mu+2} .
$$

Proof. Following the method used in $[\mathbf{5}, \mathbf{6}]$

$$
\begin{aligned}
u-v_{n} & =\sum_{k=0}^{\infty}\left\langle u, \varphi_{k}\right\rangle_{w} \varphi_{k}-\sum_{k=0}^{n} b_{k} \varphi_{k} \\
& =\sum_{k=0}^{n}\left(\left\langle u, \varphi_{k}\right\rangle_{w}-b_{k}\right) \varphi_{k}+\sum_{k=n+1}^{\infty}\left\langle u, \varphi_{k}\right\rangle_{w} \varphi_{k} .
\end{aligned}
$$

However, $\left\langle u, \varphi_{k}\right\rangle_{w}-b_{k}=\left\langle u, \varphi_{k}\right\rangle_{w}-\left\langle v_{n}, \varphi_{k}\right\rangle_{w}=\left\langle u-v_{n}, \varphi_{k}\right\rangle_{w}$. By the Cauchy-Schwarz inequality and Theorem 4.1, $\left\langle u-v_{n}, \varphi_{k}\right\rangle_{w} \leq$ $\left\|u-v_{n}\right\|_{w}|| \varphi_{k} \|_{w} \leq c_{1} n^{-r+\nu+1}$. Also, $\left|\left\langle u, \varphi_{k}\right\rangle_{w}\right| \leq c_{2} n^{-r}[\mathbf{6}], n \geq r$. Thus, for $n \geq r$,

$$
\begin{aligned}
\left|u-v_{n}\right| & \leq c_{1} n^{-r+\nu+1} \sum_{k=0}^{n}\left\|\varphi_{k}\right\|_{\infty}+\sum_{k=n+1}^{\infty} c_{2} n^{-r+\nu+\mu} \\
& \leq c_{3} n^{-r+\nu+1} \cdot n^{\mu+1}+c_{4} n^{-r+\mu+1} \\
& \leq c n^{-r+\nu+\mu+2}
\end{aligned}
$$

so that $\left\|u-v_{n}\right\|_{\infty}=0\left(n^{-r+\nu+\mu+2}\right)$, and $u_{n} \rightarrow u$ if $r>\nu+\mu+2$.

Example 4.3. In Example $4.1 \nu=0, \mu=1 / 2$ [15], so that $v_{n}$ converges uniformly to $u$ if $r>5 / 2$ and $\left\|u-v_{n}\right\|_{\infty}=0\left(n^{-r+5 / 2}\right)$. 
Example 4.4. In Example $4.2 \nu=0$ and $\mu=0$ since $\left\|t_{n}(x)\right\|_{\infty}=1$. Thus, $\left\|u-v_{n}\right\|_{\infty}=0\left(n^{-r+2}\right)$.

Note that the convergence rate here is somewhat worse than the known $0\left(n^{-r} \log n\right)$ convergence for $u_{n}$.

Example 4.5. We note that the convergence rates given in Theorem 4.1 and 4.2 are not optimal. For instance, if we use Gaussian quadrature with $n+1$ nodes in Examples 4.1 and 4.3 then, arguing as in [3], it can be shown that $v_{n}$ is the Lagrange interpolant of the solution $\left\{z_{k}\right\}$ to the Nyström equations

$$
z_{k}=f\left(t_{k}\right)+\sum_{j=0}^{n} w_{k} k\left(t_{k}, t_{j}\right) z_{j}, \quad 0 \leq j \leq n .
$$

Using this fact and the well-known convergence theory for the Nyström method [1], it can be shown that

$$
\left\|u-v_{n}\right\|_{\infty} \leq n^{-r+1 / 2} .
$$

If we consider only $L_{w}$ convergence, then the estimate (4.18) can be improved. Again, arguing as in [2] using the relation between $v_{n}$ and the Sloan iterate $\hat{v}_{n}=f+K_{n} v_{n}$, we have

$$
\left\|u-v_{n}\right\|_{w} \leq\left(1+\left\|\pi_{n}\right\|\right)\left\|u-p_{n}\right\|_{w}+\left\|u-\hat{v}_{n}\right\|_{w},
$$

where $\pi_{n}: C[a, b] \rightarrow L_{w}$ maps a continuous function to its Lagrange interpolant on the quadrature nodes $\left\{t_{k}\right\}_{k=0}^{n}$ and $p_{n}$ is the polynomial of best uniform approximation of degree $\leq n$ to $u$. In this case $\left\|\pi_{n}\right\| \leq c$ and $\left\|u-\hat{v}_{n}\right\|_{w} \leq c_{2} n^{r}$ since $\hat{v}_{n}$ is the Nyström interpolant of $\left\{z_{k}\right\}_{k=0}^{n}$. Using this in (4.22)

$$
\left\|u-v_{n}\right\|_{w} \leq c n^{-r}
$$

5. Conclusions. We have considered solving the Fredholm integral equation (1.1) by using Galerkin's method with orthonormal polynomial bases. When these are the Legendre polynomials and Gaussian quadrature is used to evaluate the inner products and integral transforms, the resulting discrete Galerkin method agrees with that studied 
by Miel in [11]. Here our basic result, Theorem 4.1, provides convergence rates sharpening the results in Theorem 5.2 of $[\mathbf{1}]$. For more general polynomial expansions our results seem to be new.

Extension of these results to discontinuous kernels, particularly Green's function kernels, and to nonlinear problems is of interest in view of the interesting parallel algorithms developed by Miel for solving two point boundary value problems by conversion to an equivalent integral equation [11].

\section{REFERENCES}

1. K. Atkinson, A survey of numerical methods for the solution of Fredholm integral equations of the second kind, SIAM, Philadelphia, 1976.

2. K. Atkinson and A. Bogomolny, The discrete Galerkin method for integral equations, Math. Comp. 48 (1987), 595-616.

3. C.T.H. Baker, The numerical treatment of integral equations, Oxford University Press, Oxford, England, 1976.

4. G.A. Chandler, Superconvergence of numerical solutions to second kind integral equations, Australian National University, Ph.D. Thesis, 1979.

5. L.M. Delves and T.L. Freeman, Analysis of global expansion methods: Weakly asymptotically diagonal systems, Academic Press, New York, 1981.

6. M.A. Golberg, Introduction to the numerical solution of Cauchy singular integral equations, in Numerical solution of integral equations (M.A. Golberg, ed.), Plenum Publishing Co., New York, 1990.

7. S. Joe, Discrete Galerkin methods for Fredholm integral equations of the second kind, IMA J. Numer. Anal. 7 (1987), 149-164.

8. G. Miel, Perturbed projection methods for split equations of the first kind, Integral Equations Operator Theory 8 (1985), 268-275.

9. - On the Galerkin and collocation methods for Cauchy singular integral equations, SIAM J. Numer. Anal. 23 (1986), 135-143.

10. - Parallel solution of Fredholm integral equtions of the second kind by orthogonal polynomial expansions, Appl. Numer. Math. 5 (1989), 345-361.

11. - Numerical solution on parallel processors of two-point boundaryvalue problems of astrodynamics, in Numerical solution of integral equations (M.A. Golberg, ed.), Plenum Publishing Co., New York, 1990.

12. I.H. Sloan, Improvement by iteration for compact operator equations, Math. Comp. 30 (1976), 758-764.

13. - Error analysis for a class of degenerate-kernel methods, Numer. Math. 25 (1976), 231-238.

14. A. Spence and K.S. Thomas, On superconvergence properties of Galerkin's method for compact operator equations, IMA J. Numer. Anal. 3 (1983), 253-271. 
15. G. Szëgo, Orthogonal polynomials, Amer. Math. Soc., Providence, RI, 1975.

2025 University Circle, Las Vegas, NV 89119-6051 